\title{
PERFORMANCE ANALYSIS OF \\ KALMAN-BASED FILTERS AND PARTICLE FILTERS FOR NON-LINEAR/NON-GAUSSIAN BAYESIAN TRACKING
}

\author{
Wenjie Shu, Zhiqiang Zheng \\ College of Electro-Mechanic and Automation \\ National University of Defense Technology \\ Changsha, Hunan 410073, P.R.China \\ Email:wjshu_0407@hotmail.com and xyzheng@sohu.com
}

\begin{abstract}
In this paper, we present an overview performance analysis of Kalmanbased filters and particle filters for Non-Linear/Non-Gaussian Bayesian tracking. The simulation results show that the particle filters have superior performance than the Kalman-based filters. Although the particle filters are time consuming, but in many situations such as the low data rate, low signal-to-noise ratio situations, the superior performance is very attractive. Copyright ${ }^{\complement}$ 2005 IFAC.
\end{abstract}

Keywords: Kalman-based filters, Particle filters, Bayesian tracking

\section{INTRODUCTION}

Many problems in statistical signal processing, automatic control, applied statistics or econometrics are in nature non-linear and non-Gaussian, which can be seen as Bayesian filtering problem (Doucet, 1998). Therefor, there is a need for accurate state estimation techniques for the Bayesian filtering problems.

The well-known and widely-used algorithm to solve the problem is the extended Kalman filter (EKF). This filter is based upon the principle of linearizing all nonlinear models (i.e., process and measurement models) using Taylor series expansions so the traditional Kalman filter (KF) can be applied. Unfortunately, in practice, the use of the EKF has two well-known drawbacks. First, the linearisation can produce highly unstable filters if the assumptions of local linearity is violated. Second, the derivation of the Jacobian matrices are nontrivial in most applications and often lead to significant implementation difficulties (Julier et al., 1997). As a result, this filter may diverge.
To address these limitations, Julier and Uhlmann in (Julier et al., 1997) proposed the unscented Kalman filter (UKF), which is a straightforward extension of the unscented transformation (UT) to the recursive estimation. The UKF is a powerful nonlinear estimation technique and has been shown to give better performance than a standard EKF in a variety of applications.

However, all these methods (EKF and UKF) are approximating the true distribution as being Gaussian - this is necessary to make the math tractable. When the non-linearity is pronounced then even the best-fitting Gaussian distribution will be a poor approximation to the posterior distribution.

Another popular solution strategy for the Bayesian filtering problem is to use sequential Monte Carlo methods, also known as particle filters (Gordon et al., 1993). These methods allow for a complete representation of the posterior distribution of the states, so that any statistical estimates, such as the mean, modes, kurtosis and variance, can be 
easily computed. They can therefore, deal with any nonlinearities or distributions.

The main object of this paper is to present an overview performance analysis of the Kalmanbased filters and particle filters for non-linear and/or non-Gaussian Bayesian tracking.

The remainder of this paper is organized as follows. We begin in Section 2 with a description of the non-linear tracking problem and its optimal Bayesian solution. The Kalman-based filters including the Extended Kalman filter and Unscented Kalman filter are described in Section 3. In section 4, various particle filters such as generic particle filter, Extended Kalman particle filter and Unscented particle filter will be discussed. Finally, in Section 5, we compare the Kalman-based filters and particle filter based on a highly non-linear tracking and analyze the simulation results. Conclusions and future work will be drawn in Section 6.

\section{BAYESIAN DESCRIPTION FOR MANEUVERING TARGET TRACKING}

Many recursive estimation problems can be formulated as

$$
\begin{aligned}
\mathbf{x}_{k+1} & =\mathbf{f}\left(\mathbf{x}_{k}, \mathbf{w}_{k}\right) \\
\mathbf{y}_{k} & =\mathbf{h}\left(\mathbf{x}_{k}, \mathbf{v}_{k}\right)
\end{aligned}
$$

where $\mathbf{f}$ is a possibly non-linear function of the state $\mathbf{x}_{k} \in \Re^{n}$ and the observation $\mathbf{y}_{k} \in \Re^{m}$ is often a non-linear mapping of the current state. Both the system model and the measurement model are inaccurate, due to modeling and/or sensor errors. This is described by the stochastic processes $\mathbf{w}_{k}$ and $\mathbf{v}_{k}$. Then the objective of tracking is to recursively estimate and predict the state $\mathbf{x}_{k}$ using the observations $\mathbf{y}_{1: k}=\left\{\mathbf{y}_{i}\right\}_{i=1}^{k}$ up to and including time $k$.

From a Bayesian perspective, the tracking problem is required to construct the pdf $p\left(\mathbf{x}_{k} \mid \mathbf{y}_{1: k}\right)$, given the data $\mathbf{y}_{1: k}$ up to time $k$. It is assumed the signals are independent with probability densities $p\left(\mathbf{w}_{k}\right), p\left(\mathbf{v}_{k}\right)$, and the initial pdf, $p\left(\mathbf{x}_{0} \mid \mathbf{y}_{0}\right) \equiv$ $p\left(\mathbf{x}_{0}\right)$, of the state vector, also known as the prior, is available ( $\mathbf{y}_{0}$ being the set of no measurements) and independent. Then, in principle, the pdf $p\left(\mathbf{x}_{k} \mid \mathbf{y}_{1: k}\right)$ may be obtained recursively in two stages: time update in (3) and measurement update in (4).

$$
\begin{aligned}
p\left(\mathbf{x}_{k+1} \mid \mathbf{y}_{1: k}\right) & =\int_{\Re^{n}} p\left(\mathbf{x}_{k+1} \mid \mathbf{x}_{k}\right) p\left(\mathbf{x}_{k} \mid \mathbf{y}_{1: k}\right) d \mathbf{x}_{k}(3) \\
p\left(\mathbf{x}_{k} \mid \mathbf{y}_{1: k}\right) & =\frac{p\left(\mathbf{y}_{k} \mid \mathbf{x}_{k}\right) p\left(\mathbf{x}_{k} \mid \mathbf{y}_{1: k-1}\right)}{p\left(\mathbf{y}_{k} \mid \mathbf{y}_{1: k-1}\right)}
\end{aligned}
$$

These equations can easily be derived using the Markov property, Bayes's rule and some standard calculations from probability theory.

The recurrence relations (3) and (4) form the basis for the optimal Bayesian solution. This recursive propagation of the posterior density is only a conceptual solution in that in general, it cannot be determined analytically.

\section{KALMAN-BASED FILTERS}

The traditional methods to deal with the Bayesian filtering problem is the Kalman filter. Howerver, in many situations of interest, the linear Gaussian assumptions of Kalman filter do not hold. The Kalman filter cannot therefore be used directly. In this section, we will introduce two Kalmanbased filters - Extended Kalman filter (EKF) and Unscented Kalman filter (UKF), which have been widely used in signal processing, automatic control, etc,.

\subsection{Extended Kalman Filter}

If (1) and (2) are non-linear, then a local linearization of the equations may be a sufficient description of the non-linear. The extended Kalman filter (EKF) is based upon this approximation. These approximations, however, can introduce large errors in the true posterior mean and covariance of the transformed (Gaussian) random variable, which may lead to sub-optimal performance and sometimes divergence of the filter.

The EKF utilizes the first term in a Taylor expansion of the non-linear function. A higher order EKF that retains further terms in the Taylor expansion exists, but the additional complexity has prohibited its widespread use.

\subsection{Unscented Kalman Filter}

An alternative to the EKF is to use the unscented Kalman filter (UKF), which was first proposed by Julier and Uhlmann (Julier et al., 1997). It builds on the principle that it is easier to approximate a Gaussian distribution than it is to approximate an arbitrary nonlinear function or transformation (J.K.Uhlmann, 1994). This method bares a superficial resemblance to Monte Carlo-type methods, but uses a deterministic sampling approach to capture the true mean and covariance estimates with a minimal set of carefully chosen sample points, which are called sigma points (Wan et al., 2000a).

For linear functions, the UKF is equivalent to the $\mathrm{KF}$. The computational complexity of the UKF is 
the same as the EKF, but it is more accurate and does not require the derivation of any Jacobians.

\section{PARTICLE FILTERS}

We have so far presented two nonlinear filtering methods that rely on Gaussian approximation. In this section, we shall present the sequential Monte Carlo method, or particle filter that does not require this assumption. The particle filter was first suggested by Gordon et al.(Gordon et $a l ., 1993)$ and the key idea is to represent the required posterior density function (pdf) by a set of random samples with associated weights and to compute estimates based on these samples and weights. As the number of samples becomes very large, this Monte Carlo characterization becomes an equivalent representation to the usual functional description of the posterior pdf, and the particle filter approaches the optimal Bayesian estimate.

In this section, we first introduce the Sequential Importance Sampling (SIS) Algorithm. Then we present the extended Kalman particle filter (EPF) and unscented particle filter (UPF), which are on the base of the particle filter.

\subsection{The Sequential Importance Sampling Algorithm}

In order to develop the details of the algorithm, let $\left\{\mathbf{x}_{0: k}^{i}, w_{k}^{i}\right\}_{i=1}^{N_{s}}$ denote a Random Measure that characterizes the posterior pdf $p\left(\mathbf{x}_{0: k} \mid \mathbf{y}_{1: k}\right)$, where $\left\{\mathbf{x}_{0: k}^{i}, i=1, \ldots, N_{s}\right\}$ is a set of support points with associated weights $\left\{w_{k}^{i}, i=1, \ldots, N_{s}\right\}$ and $\mathbf{x}_{0: k}=\left\{\mathbf{x}_{j}, i=1, \ldots, k\right\}$ is the set of all states up to time $k$. the weights are normalized such that $\sum_{i} w_{k}^{i}=1$. Then, the posterior density at $k$ can be approximated as

$$
p\left(\mathbf{x}_{0: k} \mid \mathbf{y}_{1: k}\right) \approx \sum_{i=1}^{N_{s}} w_{k}^{i} \delta\left(\mathbf{x}_{0: k}-\mathbf{x}_{0: k}^{i}\right)
$$

We therefore have a discrete weighted approximation to the true posterior, $p\left(\mathbf{x}_{0: k} \mid \mathbf{y}_{1: k}\right)$. The weights are chosen using the principle of Importance Sampling (Bergman, 1999) (Doucet, 1998). This principle relies on the following: Suppose $p(x) \propto \pi(x)$ is a probability density from which it is difficult to draw samples, but for which $\pi(x)$ can be evaluated.

So, if the samples, $\mathbf{x}_{0: k}$, were drawn from an importance density, $q\left(\mathbf{x}_{0: k}\right)$ then the weights in (5) are defined to be

$$
w_{k}^{i} \approx \frac{p\left(\mathbf{x}_{0: k}\right)}{q\left(\mathbf{x}_{0: k}\right)}
$$

If the importance density is chosen to factorise such that

$q\left(\mathbf{x}_{0: k} \mid \mathbf{y}_{1: k}\right)=q\left(\mathbf{x}_{k} \mid \mathbf{x}_{0: k-1}, \mathbf{y}_{1: k}\right) q\left(\mathbf{x}_{0: k-1} \mid \mathbf{y}_{1: k-1}\right)(7)$

and the weight update equation can then be shown to be

$$
w_{k}^{i}=w_{k-1}^{i} \frac{p\left(\mathbf{y}_{k} \mid \mathbf{x}_{k}^{i}\right) p\left(\mathbf{x}_{k}^{i} \mid \mathbf{x}_{k-1}^{i}\right)}{q\left(\mathbf{x}_{k}^{i} \mid \mathbf{x}_{0: k}^{i}, \mathbf{y}_{1: k}\right)}
$$

Particle filters rely on sequential important sampling (SIS) and, as a result, require the design of proposal distributions that can approximate the posterior distribution reasonably well. In general, it is hard to design such proposals. The easiest and common strategy is to sample from the prior distribution and to weight the particles according to the measurement likelihood. An alternative is to sample from the measurement likelihood. These strategies can, however, fail if the new measurements appear in the tail of the prior or if the likelihood is too peaked in comparison to the prior. Various other approaches have been proposed to solve this problem, such as prior editing, rejection methods and auxiliary particle filters. These three methods are all suffered from the numerous inefficiencies. However, it is possible to construct suboptimal approximations to the optimal importance density by using local linearization techniques (Doucet, 1998). Such linearizations use an importance density that is a Gaussian approximation to $p\left(\mathbf{x}_{k} \mid \mathbf{x}_{k-1}, \mathbf{y}_{k}\right)$. Another approach is to estimate a Gaussian approximation to $p\left(\mathbf{x}_{k} \mid \mathbf{x}_{k-1}, \mathbf{y}_{k}\right)$ using the unscented transform (Merwe et al., 2000).

\subsection{Extended Kalman Particle Filter (EPF)}

Within the particle filter framework, a separate EKF is used to generate and propagate a Gaussian proposal distribution for each particle, i.e.,

$$
q\left(\mathbf{x}_{k}^{i} \mid \mathbf{x}_{0: k}^{i}, \mathbf{y}_{1: k}\right) \approx \mathcal{N}\left(\overline{\mathbf{x}}_{k}^{i}, \hat{\mathbf{P}}_{k}^{i}\right) \quad i=1, \ldots, N .
$$

That is, at time $\mathrm{k}-1$ one uses the EKF equations, with the new data, to compute the mean and covariance of the importance distribution for each particle. Next, we sample the $i$-th particle from this distribution. The method requires that we propagate the covariance $\hat{P}_{k}^{i}$ and specify the EKF process and measurement noise covariances.

Since the EKF is an MMSE estimator, this local linearization method leads to an improved annealed sampling algorithm, whereby the variance of each proposal distribution changes with time. Ideally, we start searching over a large region of the error surface and as time progresses, we concentrate on the regions of lower error. 


\subsection{Unscented Particle Filter (UPF)}

The unscented particle filter was developed to address some of the short-comings of the extended Kalman particle filter.

As shown in section 3.2, the unscented Kalman filter (UKF) is able to more accurately propagate the mean and covariance of the Gaussian approximation to the state distribution, than the EKF. Distributions generated by the UKF generally have a bigger support overlap with the true posterior distribution than the overlap achieved by the EKF estimates. The UKF also has the ability to scale the approximation errors in the higher tailed distributions. This makes the UKF very attractive for the generation of proposal distributions (Merwe et al., 2000).

The new filter that result from using a UKF for proposal distribution generation within the particle filter framework is called the Unscented Particle Filter (UPF), which takes advantage of the good features of both UKF and particle filters, and avoids their limitations. The UPF uses a UKF for proposal distribution within the particle filter framework. Specifically, the proposal distribution for each particle is as follows:

$q\left(\mathbf{x}_{k}^{i} \mid \mathbf{x}_{0: k-1}^{i}, \mathbf{y}_{1: k}\right)=\mathcal{N}\left(\overline{\mathbf{x}}_{k}^{i}, \hat{\mathbf{P}}_{k}^{i}\right), \quad i=1, \ldots, N(10$

where $\overline{\mathbf{x}}_{k}$ and $\hat{\mathbf{P}}_{k}$ are the mean and covariance of $\mathbf{x}$, computed using UKF. The UPF algorithm is easily obtained by plugging the UKF step and Equation (10) into the generic particle filter algorithm.

\section{SIMULATION EXPERIMENT}

Here we consider the following dynamic system as an illustrative example:

$$
\begin{aligned}
& x_{k}=f_{k}\left(x_{k-1}, k\right)+v_{k-1} \\
& y_{k}=\frac{x_{k}^{2}}{20}+n_{k}
\end{aligned}
$$

where

$f_{k}\left(x_{k-1}, k\right)=\frac{x_{k-1}}{2}+\frac{25 x_{k-1}}{1+x_{k-1}^{2}}+8 \cos (1.2(k-1))$

and $v_{k-1}$ and $n_{k}$ are zero mean Gaussian random variables with variances $Q_{k-1}$ and $R_{k}$, respectively. We use $Q_{k-1}=10$ and $R_{k}=1$. Given only the noisy observations, $y_{t}$, the different filters were used to estimate the underlying clean state sequence $x_{t}$ for $t=1, \ldots, T$. Here $T=60$. This example has been analyzed before in many publications (Carlin et al., 1992) (Gordon et al., 1993) (Arulampalam et al., 2002).

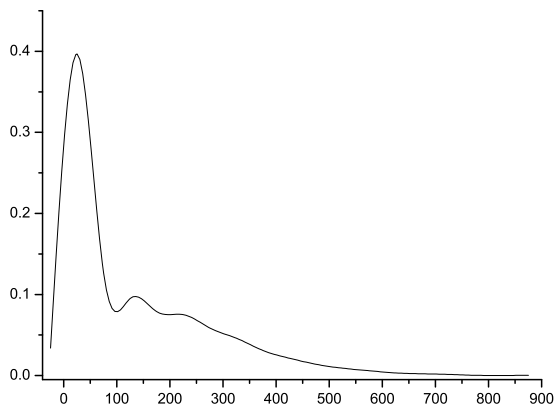

Fig. 1. pdf of likelihood $p\left(y_{k} \mid x_{k}\right)$

Table 1. RMSE for 100 Monte Carlo simulations with Kalman-based filters

\begin{tabular}{|c|c|c|c|}
\hline \multirow[t]{2}{*}{ Algorithm } & \multicolumn{2}{|c|}{ RMSE } & \multirow{2}{*}{$\begin{array}{l}\text { Execution time } \\
(\mathrm{s})\end{array}$} \\
\hline & mean & var & \\
\hline EKF & 102.4464 & 82.9801 & 0.0369 \\
\hline UKF & 7.6539 & 11.2789 & 0.0335 \\
\hline SR-UKF & 7.2869 & 6.9294 & 0.0342 \\
\hline $\mathrm{PF}$ & 5.4149 & 2.5382 & 1.0812 \\
\hline $\mathrm{EPF}$ & 5.1272 & 2.5541 & 6.3639 \\
\hline UPF & 4.8438 & 2.9322 & 2.8613 \\
\hline SR-UPF & 4.7998 & 2.4346 & 4.0925 \\
\hline
\end{tabular}
and particle filters

This is highly nonlinear in both the process and observation equations. Notice the term in the process equation which is independent of $x_{k}$ but varies with time $k$, which can be interpreted as time varying noise. The likelihood $p\left(y_{k} \mid x_{k}\right)$ in Fig. 1 is very peaked and has a heavy tail, which makes the problem more difficult to address using conventional methods.

In Table 1, the Root Mean Square Error (RMSE) and the Execution time ${ }^{1}$ of the algorithms for Kalman-based filters and particle filters are compared. The RMSE is defined as follows:

$$
R M S E=\sqrt{\frac{1}{T} \sum_{k=1}^{T} \frac{1}{N_{m c}} \sum_{i=1}^{N_{m c}}\left(\hat{x}_{k}^{i}-x_{k}^{i}\right)^{2} .}
$$

The SR-UKF denotes the square root unscented Kalman filter, which was proposed in (Merwe et al., 2001). And SR-UPF is the square root unscented particle filter.

The example was repeated $N_{m c}=100$ times independently with random re-initialization for each run. So here we adopt the mean and variance of RMSE. All of the particle filters used $N=500$ particles.

\subsection{Kalman-Based Filters}

The extended Kalman filter's local liberalization and Gaussian approximation are not a sufficient

\footnotetext{
1 which is a relative measurement of algorithms.
} 


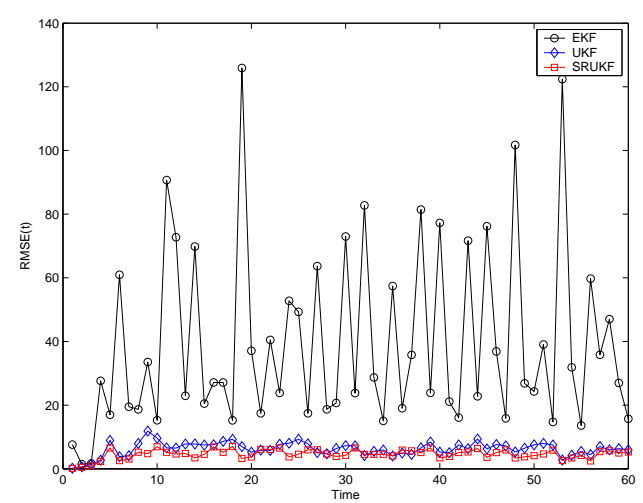

Fig. 2. RMSE(k) for different Kalman-based filters description of the non-linear and non-Gaussian nature of the example. This can be seen from Fig. ??. The mean of the filter is rarely close to the true state.

The RMSE measure in Table 1 indicates EKF erros is the least accurate of the algorithms at approximating the posterior. The approximations made by the EKF are inappropriate in this example.

The improvement in RMSE performance of the UKF over that of the EKF is great which can be seen in Table 1. And the superior performance is also well documented (Kitagawa, 1996) (Julier et al., 1997) (Wan et al., 2000a) (Wan et al., 2000b). SR-UKF has a little improvement in performance over UKF for RMSE.

In Fig. 2, the RMSE is presented for each time, i.e., according to the equation (14) for the different Kalman-Based methods. The UKF and SR-UKF are much more stable than the EKF, which is an attractive factor in the application.

$$
\operatorname{RMSE}(k)=\sqrt{\frac{1}{N_{m c}} \sum_{i=1}^{N_{m c}}\left(\hat{x}_{k}^{i}-x_{k}^{i}\right)^{2} .}
$$

\subsection{Particle Filters}

The RMSE errors in Table 1 indicate that, in highly non-linear environments, the particle filters offers an improvement in performance over an EKF and UKF. This improvement results from approximating the density rather than the models.

When using a particle filter, one can often expect and frequently achieve an improvement in performance by using far more particles or alternatively by employing EPF or UPF. An importance density tuned to a particular problem will yield an appropriate trade off between the number of particles and the computational expense necessary for each particle, giving the best qualitative performance with affordable computational effort.

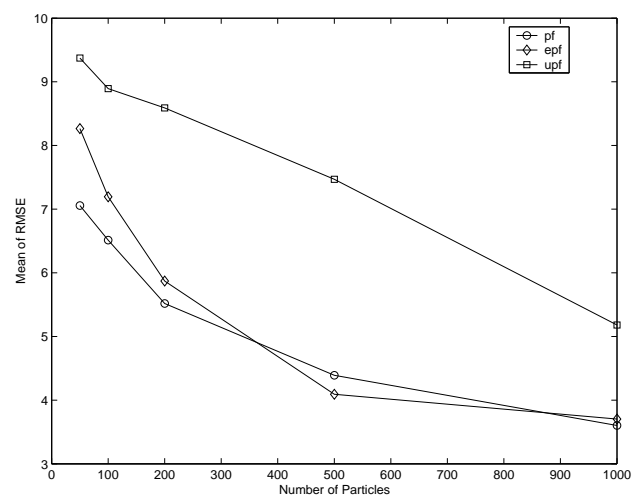

Fig. 3. particle filters with different number of particles

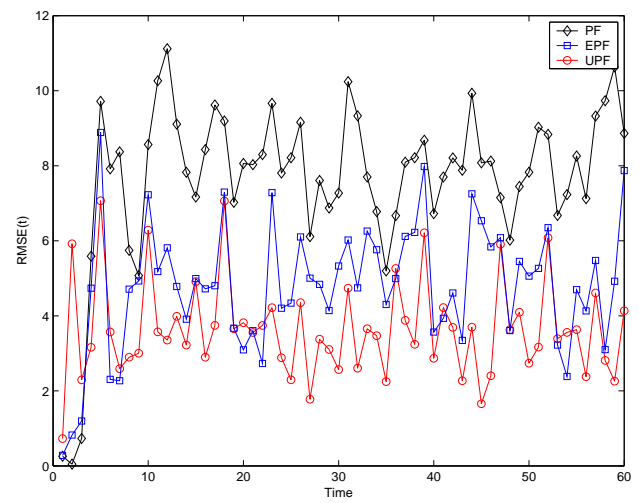

Fig. 4. RMSE(k) for different particle filters

Fig. 3 shows performance of $\mathrm{PF}, \mathrm{EPF}$ and $\mathrm{UPF}$ using different number of particles: 50, 100, 200, 500. The RMSEs using particles from 50 to 500 decrease rapidly, and 1000 and beyond have little room for improvement. Particle filter using 1000 particles almost has the same RMSE with EPF and UPF using only 200 particles. So choosing the importance density to be well suited to a given application requires careful thought. The choice made is crucial.

In Fig. 4 the RMSE is presented for each time, i.e., according to the equation (14) for the different particle filters.

\section{CONCLUSIONS AND FUTURE WORK}

In the simulation study in section 5, the particle filters improved the tracking performance compared to the Kalman-based filters for nonlinear/non-Gaussian Bayesian tracking. However, particle filters can be time consuming if many particles are used.

For Kalman-based filters, the UKF has superior performance over the EKF with the same computation time, and SR-UKF has a little improvement in performance than UKF. The UKF and SRUKF are much more stable than the EKF. When using a particle filter, one can often expect and fre- 
quently achieve an improvement in performance by using far more particles or alternatively by employing EPF or UPF. On the whole, the particle filter is superior to the Kalman-based filters.

Although the particle filter is time consuming, but in the low data rate, low signal-to-noise ratio situations where sensor responses may provide very ambiguous information about the state of the target, the superior performance of particle filters is very attractive.

In our future work, we will extend the range of applications of the particle filters. And the parallel particle filter and hardwarization of particle filter is also our direction of future work.

\section{REFERENCES}

A. Doucet, On Sequential Monte Carlo Mehtods for Bayesian Filtering, Technical Report, University of Cambridge, UK, Department of Engineering, 1998.

B. P. Carlin, N. G. Polson, D. S Stoffer, A Monte Carlo Approach to nonnormal and non-linear state-space modelling, Journal of the American Statistical Association, 87(418), pp. 493500, 1992.

E. A. Wan, R. van derMerwe, and A. T. Nelson, Dual Estimation and the Unscented Transformation, In: Neural Information Processing Systems 12.2000, pp. 666-672, MIT Press.

E. A. Wan and R. van der Merwe, The Unscented Kalman Filter for Nonlinear Estimation, In: Proc. of IEEE Symposium 2000 (AS-SPCC), Lake Louise, Alberta, Canada, Oct. 2000.

F. Gustafsson, F. Gunnarsson, N. Bergman, U. Forssell, J. Jansson, R. Karlsson, and P. J. Nordlund, Particle filters for positioning, navigation and tracking. IEEE Transactions on Signal Processing, Feb, 2002.

G. Kitagawa, Monte Carlo Filter and Smoother for Non-Gaussian Non-linear State Space Models. Journal Of Computational and Graphical Statistics, Vol. 5(1), pp. 1-25, 1996.

J. K. Uhlmann. Simultaneous map building and localization for real time applications. Technical report, University of Oxford, 1994. Transfer thesis.

M. Arulampalam, S. Maskell, N. Gordon, T. Clapp, A Tutorial on Particle Filters for On-line Nonlinear/Non-Gaussian Bayesian Tracking, IEEE Transactions on Signal Processing, Vol. 50, Num. 2, pp. 174-189, February 2002 .

N. Bergman, Recursive Bayesian Estimation: Navigation and Tracking Applications, PhD Thesis, Linkoping University, Sweden, 1999.

N. Gordon, D. Salmond, A. F. M. Smith, Novel Approach to Non-linear and Non-
Gaussian Bayesian State Estimation. IEEE Proceeding-F, Vol. 140, pp. 107-113, 1993.

R. van der Merwe and E. A. Wan, The SquareRoot Unscented Kalman Filter for State and Parameter-Estimation, In: International Conference on Acoustics, Speech, and Signal Processing, Salt Lake City, Utah, May, 2001

R. van der Merwe, A. Doucet, N. de Freitas and E. Wan, The Unscented Particle Filter, In: Advances in Neural Information Processing Systems (NIPS13), MIT Press, Eds. T. K. Leen, T. G. Dietterich and V. Tresp, Dec, 2000

S. J. Julier and J. K. Uhlmann, A New Extension of the Kalman Filter to Nonlinear Systems, In: Proceedings of AeroSense: The 11th International Symposium on Aerospace/Defense Sensing, Simulation and Controls, 1997.

S. J. Julier, The Scaled Unscented Transformation, In: Proceedings of the American Control Conference, Vol. 6, pp. 4555-4559, 2002. 